\title{
Chapter 12 \\ Development of an Electric-Load \\ Intelligence System for Component Level \\ Disaggregation to Improve Energy \\ Efficiency of Machine Tools
}

\author{
Nitesh Sihag $\mathbb{D}$ and Kuldip Singh Sangwan $\mathbb{D}$
}

\begin{abstract}
Energy and resource efficient manufacturing has become a key priority due to higher energy cost, market competition and environmental regulations. Better transparency and higher levels of disaggregation of energy data are necessary for energy efficiency improvement of machine tools. Since the beginning of the $21 \mathrm{st}$ century, some attempts have been made by the researchers to quantify the energy data but only up to the operational state of the machine tool. Better accuracy and transparency require disaggregation up to the component level. This study proposes an Electric-Load Intelligence (E-LI) system for identification of machine tool operating state and disaggregation of time and energy consumed up to the component level. The energy profile is obtained at the power input of a machine tool and analyzed using a set of signal processing techniques and load-disaggregation algorithms. The proposed methodology is validated through a case study of milling process. Various classifiers used in the disaggregation algorithms are compared for their accuracies using the case study data. The results reveal that only a small portion of the total cutting energy $(782.24 \mathrm{~kJ})$ was used for actual material removal $(40.73 \mathrm{~kJ})$. The proposed study provides accurate data in user friendly format to assist designers and manufacturers for strategic and economic decision making.
\end{abstract}

Keywords Electric-Load intelligence $\cdot$ Non-intrusive load monitoring $\cdot$ Energy disaggregation

\footnotetext{
N. Sihag $\cdot$ K. S. Sangwan $(\varangle)$

Department of Mechanical Engineering, Birla Institute of Technology and Science,

Pilani-Campus, Pilani 333031, RJ, India

e-mail: kss@ pilani.bits-pilani.ac.in

N. Sihag

e-mail: P2014009@pilani.bits-pilani.ac.in

(C) The Author(s) 2020

K. S. Sangwan and C. Herrmann (eds.), Enhancing Future Skills and Entrepreneurship,

Sustainable Production, Life Cycle Engineering and Management,

https://doi.org/10.1007/978-3-030-44248-4_12
} 


\subsection{Introduction}

Energy efficiency of machine tools has become an important aspect of energy saving studies due to high energy consumption and low efficiency of machine tools. Also, electric energy consumption by machine tools is responsible for more than $99 \%$ of their carbon emissions (Li and Kara 2011). Hence, any reduction in energy consumption by machine tools will lead to economic as well as environmental benefits. During the early 21 st century, the crude oil prices experienced an exponential surge from approximately 25 USD per barrel in 2001 to approximately 100 USD per barrel in 2008. This heightened the need for energy saving; and energy consumption emerged as an important criterion for modeling and optimization studies (Camposeco-Negrete 2015; Kant and Sangwan 2014; Zhang et al. 2018). The research for the improvement of surface finish and tool life, and reduction of cutting forces by optimizing cutting parameters is going on for more than 100 years since the era of Taylor equation. The optimization of cutting parameters is confined to saving of cutting energy, which consists of less than $15 \%$ of total energy demand of machine tools. The remaining $85 \%$ of the energy is consumed as fixed energy by the auxiliary components (Sihag and Sangwan 2019) (coolant pump, axis feed motors, chip conveyor, spindle motor, router, fans, lights, lubricant pump, etc.). Therefore, analysis and reduction of fixed energy of the machine tools gained attention in the recent years (Jia et al. 2017; Mustafaraj et al. 2015; Li et al. 2011). The modeling and improvement strategies for cutting and fixed energy have been studied in recent years.

The studies have emphasized that efficient monitoring and transparency in energy flow of machine tools is a primary prerequisite for energy saving (Teiwes et al. 2018). It has been observed that energy assessment at each hierarchical level is essential to support efficient decision making for energy saving (Vikhorev et al. 2013). Therefore, innovative and effective energy monitoring and management approaches are required to promote energy efficient machining. Some attempts have been made towards energy data acquisition, mapping and analysis to save energy (Zein et al. 2011). A few studies have provided generic models for estimation of machine tool energy consumption using mathematical modeling and finite elemental analysis (Pervaiz et al. 2015; Huang et al. 2016). The correlation between the energy consumption and $\mathrm{NC}$ codes has been analyzed to obtain a better picture of energy consumption by various components of a machine tool (He et al. 2011). Micro analysis of the machining energy has been provided to quantify the energy consumption up to component and activity levels for better energy transparency (Sihag and Sangwan 2019). Event stream mapping has been used as an efficient approach for automated energy monitoring of the machine tools (Vijayaraghavan and Dornfeld 2010). But the application of intelligent techniques for energy disaggregation is hardly addressed. A few studies have used various machine learning approaches for determining the operating state of a machine tool (O'Driscoll et al. 2015; Sihag et al. 2018). The energy data is processed and classified into pre-defined classes using various classifiers such as support vector machine (SVM), tree classifier, k-nearest neighbor (k-nn), etc. 
It is still a challenge for researchers to identify where and how the energy is consumed during a machining process due to complex structure of the machine tools and large number of energy consuming components. So far, the studies have been confined to the identification of the operational state of the machine tools. The existing research does not disaggregate the energy demand up to the component level to make it more transparent and easy to identify the focused measures for energy efficiency. To bridge this gap, the current study. The current study proposes a non-intrusive Electric-Load Intelligence (E-LI) system using a combination of load disaggregation algorithms (such as feature extraction, support vector machines, median absolute deviation etc.) to estimate the energy consumption and operational time for each machining state as well as for energy consuming components of a machine tool. The main contributions of the current study can be summarized as:

- Machine tool status identification using an Electric-Load-Intelligence system.

- Disaggregation of the energy consumption of machine tools up to the component level.

- Comparison of the accuracies of various classifiers used in the literature.

\subsection{Methodology}

\subsubsection{Electric-Load Intelligence Concept}

Electric-Load Intelligence (E-LI) is defined as a detailed analysis of energy consumption behavior of electric appliances and development of intelligent applications using structured algorithms to promote efficient use of electricity (Cheng et al. 2006). The proposed E-LI system serves as a novel solution for the widely discussed issue of industrial smart metering (Herrmann et al. 2011). It can be classified as a blind identification problem, where the energy consumption of individual components is identified using the aggregate data of the machine tool measured at the main power input. In general, the operating states of machine tools are classified as idle state, machine ready state and cutting state. The current study proposes a two-tier classification technique. In the first step, the energy and time consumption for each machining state is determined. In the next step, the energy and time consumed by each energy-consuming component in each machining state is calculated.

Mathematically, the power consumption of a machine tool can be represented as

$$
P(t)=\sum_{i=1}^{n} P_{t}^{i}+\varepsilon_{t}
$$


where $P(t)$ is the power consumed by machine tool at time $t, P_{t}^{i}$ is the power drawn by component $i$ at time $t, n$ is number of energy consuming components and $\varepsilon_{t}$ is the measurement error.

The main objective of the proposed algorithm is to decompose the total power consumption by the machine tool into component specific power signals in order to achieve disaggregated energy sensing. Each electrical component used in machine tools has a unique energy consumption pattern known as load signature. This signature is used to identify the specific component activation in the total power signature of the machine tool. The framework of the methodology used to develop the intelligent non-intrusive load monitoring (NILM) algorithm in the present study is presented in Fig. 12.1.

The power drawn by the machine tool is measured at the main supply input to obtain root mean square (RMS) power waveform. The activation of an event is identified using an event detection algorithm. A set of features is extracted for each event and compared against the feature data stored in the system library of trained classifiers. A series of conditional statements are applied to calculate the energy consumption and duration for each operational state and auxiliary components.

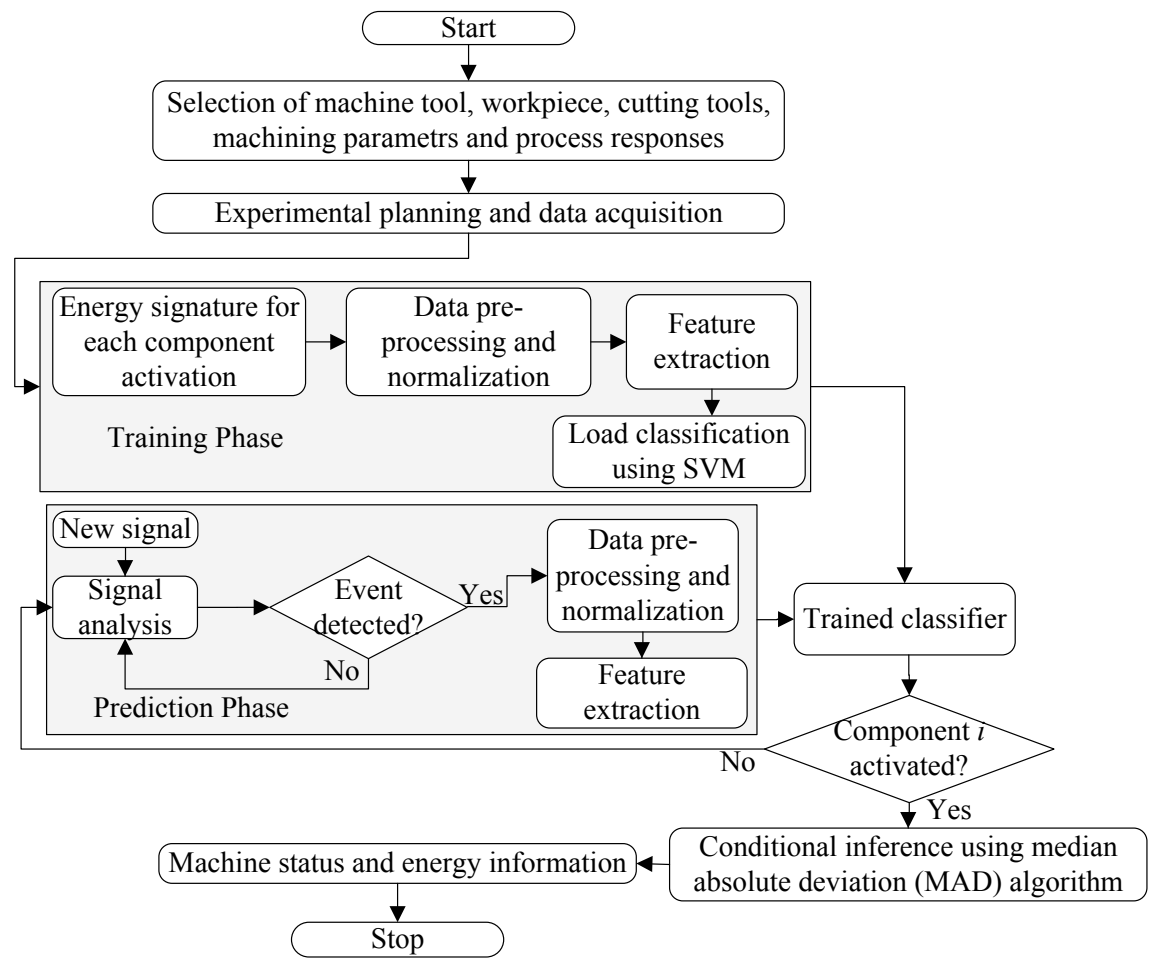

Fig. 12.1 The research methodology adopted for the present study 


\subsubsection{Load Signature Acquisition and Preprocessing}

This study uses a supervised learning process to train the proposed E-LI system. The system is trained with a known data set labelled with a predefined class. For training the algorithm, the power signature for the major energy consuming components including spindle activation at different RPMs, activation of axis motors in $\mathrm{x}, \mathrm{y}$, and $\mathrm{z}$ directions at cutting and rapid speeds, rotation of automatic tool changer, activation of coolant pump, and basic module are recorded. The energy measurement device is installed at the main power input and load signature is recorded for each component activation for five times. The captured raw data is then processes with a series of data processing and conditioning techniques including filtering and normalization to reduce the noise, standardize the data and compensate for any power quality related issues.

\subsubsection{Feature Extraction}

The original recorded power signatures are raw waveforms with large dimensions and, generally not suitable for use by the classifiers. In practice, a finite set of measurements called as features are extracted from the raw waveform for each signal segment. The objective of feature extraction is to decrease the dimension of the raw signal while maintaining the required information. The bottom line to choose appropriate features is that they should be able to capture the similarities among the signals from the similar classes and differences among the signals from the different classes. In the present study, six unique features are extracted for each event activation. These features have been selected from literature on pattern recognition studies. They are later used by the different classification algorithms to assign class to a set of extracted features.

\subsubsection{Load Disaggregation}

In this step, activation of a component is classified using a known database of component power signatures and extracted features. Various classifiers have been used in the literature to classify the load signatures such as k-nn, SVM, Tree algorithm, etc. The selection of a suitable classifier depends on its performance and ability to classify the feature data into respective classes accurately. The present study compares the accuracy of these classifiers. SVM is selected as the suitable classifier for high accuracy as explained in Sect. 12.3. It is an efficient classification approach for high dimensional data and exhibits good performance in general (Widodo and Yang 2007). It uses a mapping function to project the non-linearly separable $\mathrm{N}$-dimensional input vector $\mathrm{x}$ into a $\mathrm{K}$-dimensional feature space $(\mathrm{K}>\mathrm{N})$ where data are linearly distinguishable. 


\subsubsection{Conditional Inference for Detection of Material Removal}

Once the system is trained, it should be able to detect and classify any unknown/new load signature. As supervised learning approach covers only the predefined data classes, therefore, additional statements are required for identification of metal cutting process. Once the proposed E-LI system has identified and classified the spindle activation, machine tool is considered to be in the machine ready state. The system then searches for a rise in power consumption caused by the commencement of material removal. In the present study, median absolute deviation (MAD) algorithm is used to identify significant rise in power. At each point of time, the median is calculated and compared to previous value and cutting is identified when the change in median is higher than a predefined threshold. Once, the cutting operation is identified, the duration and energy consumption of machine ready and cutting state is calculated. Once trained, the algorithm can be run for any unknown/new data to predict the most likely class for the new data.

\subsection{Experimental Validation of the Proposed E-LI System}

\subsubsection{Experimental Set-Up}

The proposed E-LI system was verified on a 3-axis CNC vertical milling center (LMW KODI 40). A cuboidal aluminum block with dimensions $70 \times 70 \times 65 \mathrm{~mm}^{3}$ was used as the test work piece. Multi-pass milling and drilling operations were performed for experimental validation of the proposed E-LI system. First, a layer of $3 \mathrm{~mm}$ thickness was removed from the surface using a $25 \mathrm{~mm}$ face mill. The cutting parameters selected for facing operation were: spindle speed $=2000 \mathrm{RPM}$, feed in $\mathrm{x}$-direction $=50 \mathrm{~mm} / \mathrm{min}$ and feed in z-direction $=100 \mathrm{~mm} / \mathrm{min}$. Next, a hole ( $\Phi 7 \mathrm{~mm} \times 30 \mathrm{~mm}$ depth) was drilled at the work piece center using $7 \mathrm{~mm}$ drill. The cutting parameters selected for drilling operation were: spindle speed $=1500$ RPM, feed in $\mathrm{x}$-direction $=50 \mathrm{~mm} / \mathrm{min}$ and feed in $\mathrm{z}$-direction $=100 \mathrm{~mm} / \mathrm{min}$.

\subsubsection{Training Phase}

The energy meter was installed at the main energy supply of the machine tool. Fluke 435 series ii, three-phase power quality and energy analyzer was used to measure the energy. The key energy consuming components identified for the VMC are spindle activation at different RPM, activation of axis motors in $\mathrm{x}, \mathrm{y}$ and $\mathrm{z}$ directions at cutting and rapid speeds, rotation of automatic tool changer, activation of coolant pump, and basic module. The power data for each component was recorded and 
stored for training of the algorithm. The power ratings for each component are also computed and recorded for energy calculations of individual components.

The recorded data is first filtered to reduce noise followed by normalization. A set of six features are obtained for the normalized signature of each activation and labelled. This data is used for training of the classifiers. In the present study, the classifiers used to classify the data are identified from the literature and their accuracy analysis is presented in Sect. 12.4.

\subsubsection{Prediction Phase}

Once the algorithm is trained and validated, it is able to detect and classify any new event activation. The proposed sensor starts to search for event activation using event detection algorithm. The system calculates the profile slope at each point and identifies an activation when slope rises beyond a predefined value $k_{1}$. The event is considered to be active until the slope of power profile decreases beyond a predefined threshold $k_{2}$. The power RMS values should also satisfy certain conditional statements to signify reduction in power consumption. The threshold slope values and power RMS conditions are determined based on preliminary experimentation and training. Once an event is identified, the power profile for that event is normalized. Six predefined features are calculated for the normalized profile and the event is classified using SVM classifier. The system uses the same approach to detect and classify each event during the milling process. After the coolant pump and spindle motor activation is identified and the system becomes stable, it looks for a rise in power consumption due to initiation of material removal process. Once the cutting starts, it keeps on recording the data until the cutting stops and power decreases beyond a predefined value. Similarly, the system records the start and stop of each component and machining state for the entire profile. A set of conditional statements are then used to calculate the energy consumption by each component and operational state. The power profile obtained for the multi pass face milling and drilling operations is shown in Fig. 12.2. The proposed algorithm detects two spindle activations, two coolant activations, and one tool change during the test run as shown in Fig. 12.2. It also identifies the machine ready and cutting states for the machine tool and quantifies the time and energy consumed by them. The total energy consumed for the machining process is hence divided into energy consumed in each machining state and further divided into energy consumed by each component in each state. Similarly, the time duration of machining process is disaggregated.

\subsection{Accuracy Analysis of the Classifiers}

Accuracy of a classification algorithm is an important criterion in selection of suitable classifier. In the present study, supervised learning data is used to obtain and compare the accuracies of different classifiers (Table 12.1). It is evident here that Quadratic 


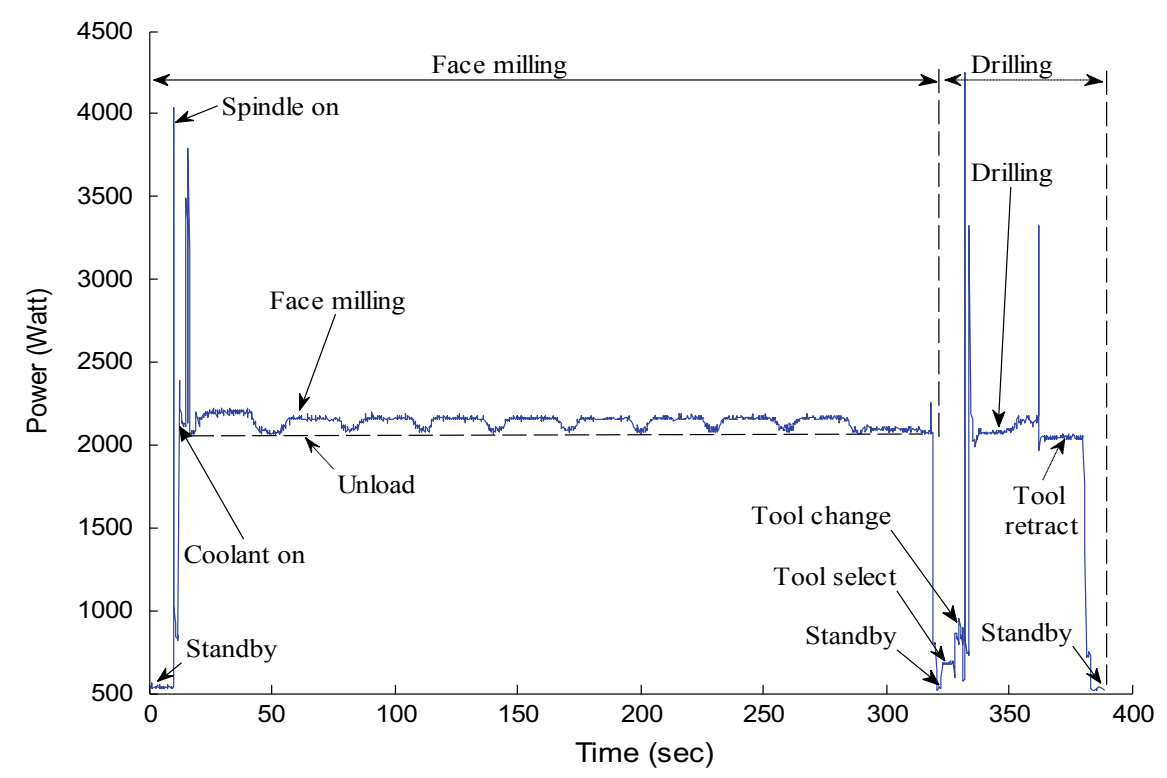

Fig. 12.2 Recorded power profile for the multi pass face milling and drilling operations

Table 12.1 Comparison of accuracy for various classifiers

\begin{tabular}{l|l|l}
\hline Classifier & Accuracy $(\%)$ & Accuracy $^{\mathrm{a}}(\%)$ \\
\hline Quadratic SVM & 92.9 & 85.9 \\
\hline Cubic SVM & 90.6 & 81.2 \\
\hline Linear SVM & 91.8 & 82.4 \\
\hline Fine Gaussian SVM & 87.1 & 82.4 \\
\hline Medium Gaussian SVM & 90.6 & 83.5 \\
\hline Coarse Gaussian SVM & 74.1 & 8.2 \\
\hline Linear discriminant & 84.7 & 83.5 \\
\hline Quadratic discriminant & 91.8 & 85.9 \\
\hline Fine k-nn & 88.2 & 78.8 \\
\hline Medium k-nn & 55.3 & 64.7 \\
\hline Weighted k-nn & 87.1 & 81.2 \\
\hline Subspace k-nn & 90.6 & 78.8 \\
\hline Subspace discriminant & 83.5 & 83.5 \\
\hline Bagged trees & 89.4 & 78.8 \\
\hline Medium tree & 64.7 & 65.9 \\
\hline
\end{tabular}

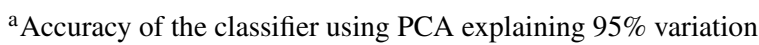


SVM exhibits the highest accuracy of $92.9 \%$. The accuracy decreases to $85.9 \%$ with principal component analysis (PCA) at $95 \%$ explained variation.

The performance of the selected classifier is further analyzed using the confusion matrix as shown in Fig. 12.3. It provides an insight of the classifier performance by recording the number of times a sample is confused to be in another class. It helps to analyze the possible reasons for errors in classification results for a large number of labeled samples and makes the required adjustments in the classifier. Confusion matrix for the present study is shown in Fig. 12.3. It is observed that FX is often confused with FY and RX is confused with RY. The reason is that the axis motors for $\mathrm{x}$ and $\mathrm{y}$ directions have the same power ratings. Once the classifier is trained, a new set of data is provided to it for validation of its performance. Data set for eight events is provided to the classifier and it is observed that the events are classified accurately, for each case, by the trained classifier.

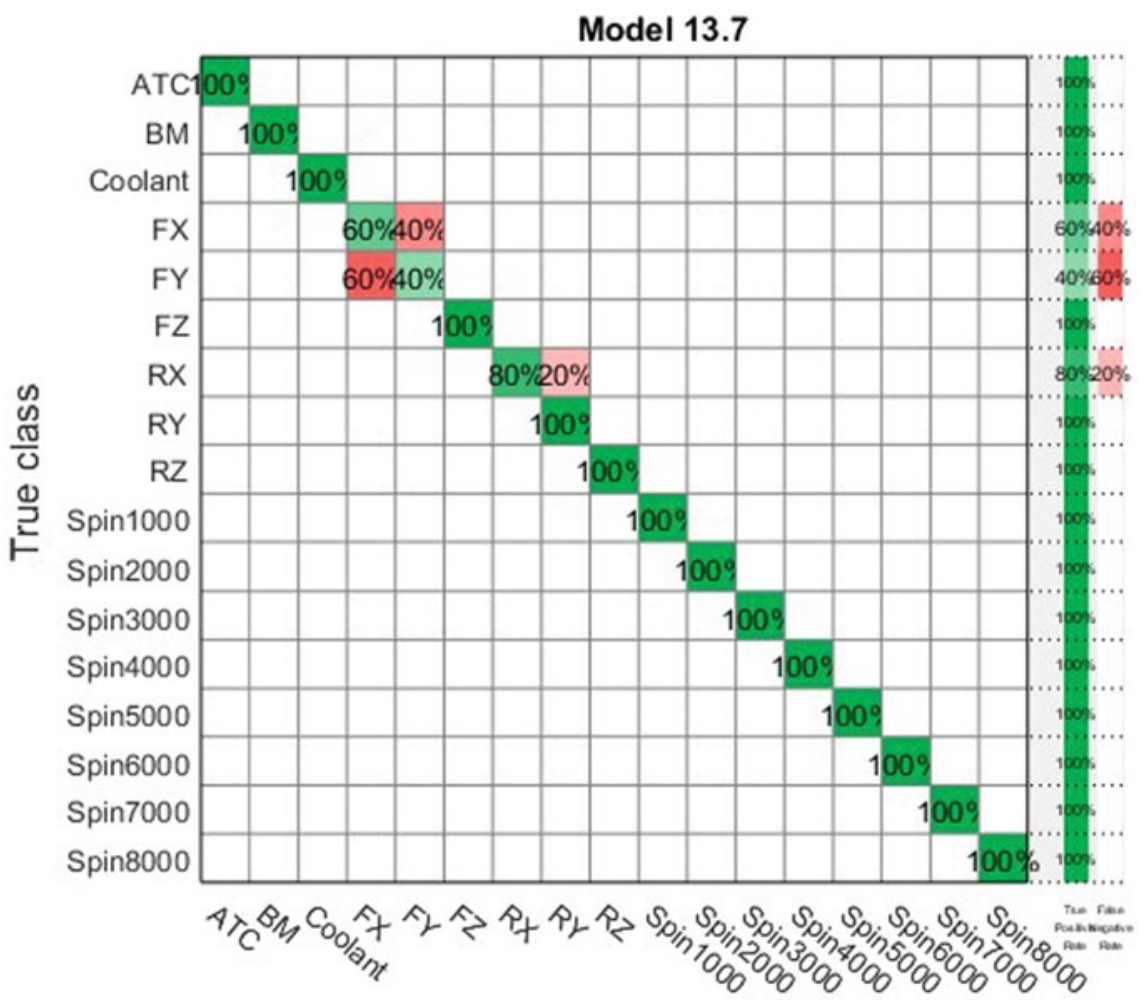

Predicted class

Fig. 12.3 Confusion matrix for SVM classifier 


\subsection{Results and Discussion}

The proposed E-LI system calculates the total energy consumed and total time duration for the milling operation as $782.24 \mathrm{~kJ}$ and $368.50 \mathrm{~s}$, respectively. The energy and time classification are shown in Figs. 12.4 and 12.5, respectively. The actual energy consumption calculated using the power profile is $782.11 \mathrm{~kJ}$. It is evident from Fig. 12.4 that $72 \%$ of the total energy is consumed during the cutting state and out of this, only $7 \%$ is consumed for actual material removal. Major percentage of the total energy is consumed by the coolant pump $(309.15 \mathrm{~kJ})$ followed by basic module $(205.91 \mathrm{~kJ})$ and spindle motor $(116.89 \mathrm{~kJ})$. The reason behind this is that a high rated motor $(1.1 \mathrm{~kW})$ is used for coolant pump and it is active for $354.5 \mathrm{~s}$. Basic module consists of the small components, which are essential for running the machine such as various relays, i/o data cards, PCBs, proximity/limit switches, lamps, etc. The power rating for basic module is less but high operation time results in higher energy consumption. It can be noted here that the present case study is conducted for a particular milling operation, hence, the idle time is negligible here. In actual industrial practices, the idle time of the machine tools is high. Hence, the energy consumed in the idle mode will be higher and the proportion of energy consumed for actual material removal will be even lesser. The disaggregated energy data provided by the proposed sensor can be used to detect the potential energy saving possibilities

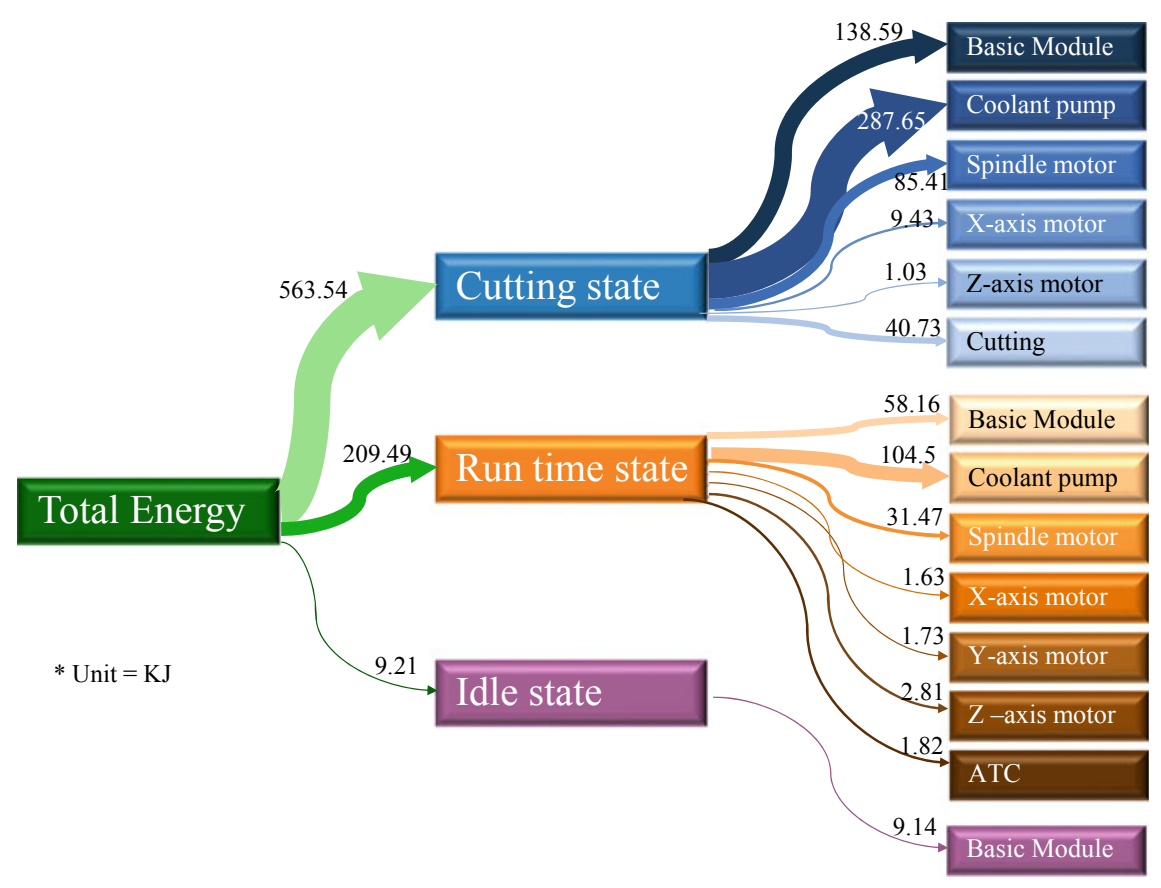

Fig. 12.4 Energy decomposition for multi pass face milling and drilling experiments 


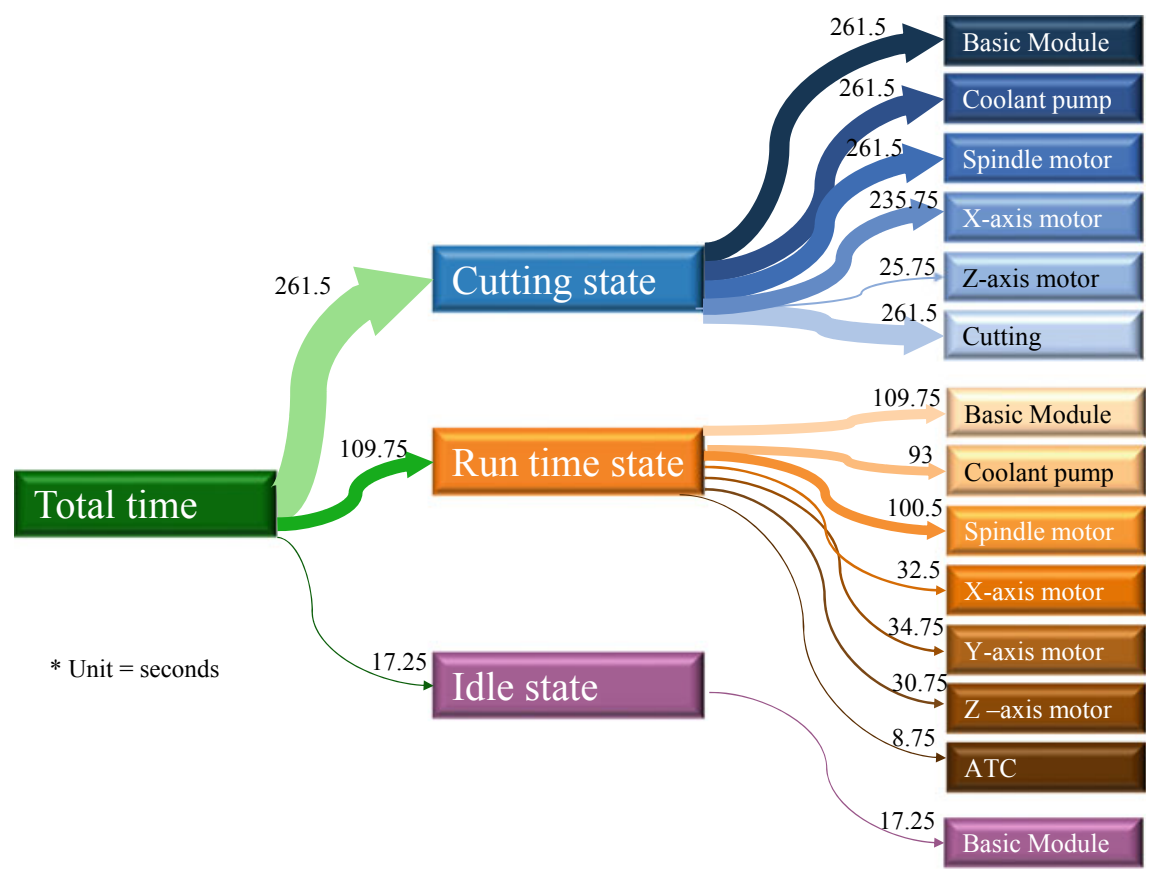

Fig. 12.5 Processing time decomposition for multi pass face milling and drilling experiments

and promote use of more efficient components for improved energy performance of the machine tools. The disaggregation up to component level provides better energy flow transparency leading to better decisions for energy efficiency.

\subsection{Conclusions}

Disaggregation of machine tool energy profile up to component level is an essential requirement to achieve better transparency in energy flow. However, it is a challenging issue for researchers due to the complexity of the machine tools. This study presents an Electric-Load Intelligence (E-LI) system for disaggregation of machine tool energy profile up to operating state and component level using a supervised learning approach. It is a non-intrusive energy monitoring method, which computes the activation time and energy consumed by each energy-consuming component by analyzing the power profile measured at the main power inlet of the machine tool. It is a low cost and viable alternative to expensive and complex multi-sensor data acquisition systems. The accuracies of different classifiers commonly used are compared and it is found that quadratic SVM classifier has maximum accuracy. The proposed methodology is verified through a milling case study. The results reveal that the total energy consumed for the test run is $782.24 \mathrm{~kJ}$, but the energy consumed for actual 
material removal is only $40.73 \mathrm{~kJ}$. The coolant pump consumes $392.15 \mathrm{~kJ}$ energy, which is almost half of the total energy consumption. The coolant pump is identified as an inefficient component here and should be replaced with a more efficient pump. It is concluded that the proposed E-LI system is an efficient and feasible approach to identify the time and energy requirement at each operating state and individual components. It provides better transparency in the energy consumption behavior of machine tools to motivate the behavioral changes leading to the reduction in energetic impacts of machining.

\section{References}

Camposeco-Negrete C (2015) Optimization of cutting parameters using response surface method for minimizing energy consumption and maximizing cutting quality in turning of AISI 6061 T6 aluminum. J Clean Prod 91:109-117

Cheng JWM, Kendall G, Leung JSK (2006) Electric-load intelligence (E-LI): concept and applications. In: ENCON 2006-2006 IEEE region 10 conference. IEEE, pp 1-4

He Y, Liu F, Wu T, Zhong FP, Peng B (2011) Analysis and estimation of energy consumption for numerical control machining. Proc Inst Mech Eng Part B J Eng Manuf 226:255-266

Herrmann C, Suh SH, Bogdanski G et al (2011) Context-aware analysis approach to enhance industrial smart metering. In: Hesselbach J, Herrmann C (eds) Glocalized solutions for sustainability in manufacturing. Springer, Berlin, Heidelberg, pp 323-328

Huang J, Liu F, Xie J (2016) A method for determining the energy consumption of machine tools in the spindle start-up process before machining. Proc Inst Mech Eng Part B J Eng Manuf 230:1639-1649

Jia S, Tang R, Lv J, Yuan Q, Peng T (2017) Energy consumption modeling of machining transient states based on finite state machine. Int J Adv Manuf Technol 88:2305-2320

Kant G, Sangwan KS (2014) Prediction and optimization of machining parameters for minimizing power consumption and surface roughness in machining. J Clean Prod 83:151-164

Li W, Kara S (2011) An empirical model for predicting energy consumption of manufacturing processes: a case of turning process. Proc Inst Mech Eng Part B J Eng Manuf 225:1636-1646

Li W, Zein A, Kara S, Herrmann C (2011) An investigation into fixed energy consumption of machine tools. In: Hesselbach J, Herrmann C (eds) Glocalized solutions for sustainability in manufacturing. Springer, Berlin, Heidelberg, pp 323-328

Mustafaraj G, Cosgrove J, Rivas-Duarte MJ, Hardiman F, Harrington J (2015) A methodology for determining auxiliary and value-added electricity in manufacturing machines. Int J Prod Res 53:5265-5277

O'Driscoll E, Kelly K, O'Donnell GE (2015) Intelligent energy based status identification as a platform for improvement of machine tool efficiency and effectiveness. J Clean Prod 105:184-195

Pervaiz S, Deiab I, Rashid A, Nicolescu M (2015) Prediction of energy consumption and environmental implications for turning operation using finite element analysis. Proc Inst Mech Eng Part B J Eng Manuf 229:1925-1932

Sihag N, Sangwan KS (2019) An improved micro analysis-based energy consumption and carbon emissions modeling approach for a milling center. Int J Adv Manuf Technol 1-17

Sihag N, Sangwan KS, Pundir S (2018) Development of a structured algorithm to identify the status of a machine tool to improve energy and time efficiencies. Procedia CIRP 69:294-299

Teiwes H, Blume S, Herrmann C, Rössinger M, Thiede S (2018) Energy load profile analysis on machine level. Procedia CIRP 69:271-276

Vijayaraghavan A, Dornfeld D (2010) Automated energy monitoring of machine tools. CIRP Ann Manuf Technol 59:21-24 
Vikhorev K, Greenough R, Brown N (2013) An advanced energy management framework to promote energy awareness. J Clean Prod 43:103-112

Widodo A, Yang BS (2007) Support vector machine in machine condition monitoring and fault diagnosis. Mech Syst Signal Process 21:2560-2574

Zein A, Li W, Herrmann C, Kara S (2011) Energy efficiency measures for the design and operation of machine tools: an axiomatic approach. In: Proceedings of the 18th CIRP international conference on life cycle engineering, Springer, Berlin, Heidelberg, pp 274-279

Zhang L, Zhang B, Bao H, Huang H (2018) Optimization of cutting parameters for minimizing environmental impact: considering energy efficiency, noise emission and economic dimension. Int J Precis Eng Manuf 19:613-624

Open Access This chapter is licensed under the terms of the Creative Commons Attribution 4.0 International License (http://creativecommons.org/licenses/by/4.0/), which permits use, sharing, adaptation, distribution and reproduction in any medium or format, as long as you give appropriate credit to the original author(s) and the source, provide a link to the Creative Commons license and indicate if changes were made.

The images or other third party material in this chapter are included in the chapter's Creative Commons license, unless indicated otherwise in a credit line to the material. If material is not included in the chapter's Creative Commons license and your intended use is not permitted by statutory regulation or exceeds the permitted use, you will need to obtain permission directly from the copyright holder.

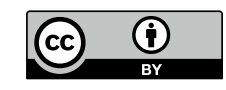

